\title{
Síndrome de veia cava superior
}

\author{
SAmuel Zuínglio de BiAsi Cordeiro ${ }^{1}$, PAUlo DE BiAsi Cordeiro²
}

A obstrução ao fluxo sanguíneo na VCS e suas manifestações clínicas têm hoje como causa principal o câncer de pulmão. A história relata que no século XVIII a sífilis e a tuberculose eram responsáveis por 40\% dos casos conhecidos. O conhecimento das alterações hemodinâmicas compreendidas nesta síndrome assim como a apuração das técnicas de diagnóstico de imagem e de citopatologia permitem hoje que o médico possa tratar de seu paciente com mais segurança e conforto do que há 10 anos. A

TC contrastada e a RM auxiliam no diagnóstico de localização da obstrução e técnicas mais antigas como a cavografia puderam ser abandonadas. O diagnóstico de obstrução da VCS e o estudo por

Doppler realizado à beira do leito em muito contribuem para a realização de procedimentos de desobstrução como a angioplastia transluminal percutânea nos casos de trombose ou estenose do vaso. Também a utilização de próteses como PTFE é de importância fundamental na condução de casos de lesão traumática da VCS durante cirurgias para câncer pulmonar ou mediastinal. No campo da radioterapia, a técnica de fracionamento permite que altas doses de irradiação sejam administradas aos pacientes portadores de neoplasias malignas, com benefícios em $70 \%$ dos casos.

(J Pneumol 2002;28(5):288-93)

\section{Superior vena cava syndrome}

Lung cancer is now the main cause of blood flow obstruction in the superior vena cava and of its clinical manifestations. History tells that in the $18^{\text {th }}$ Century, syphilis and tuberculosis were responsible for $40 \%$ of the known cases. The knowledge of hemodynamic changes seen in this syndrome and the improvement of diagnostic and cytopathologic techniques allow for a safer and more comfortable treatment of the patient than 10 years ago. Contrast CT and MR added to the identification and location of the obstruction, and older techniques such as cavography could be abandoned. SVC obstruction diagnosis and Doppler studies carried out at the bed of the patient contribute to deobstruction procedures such as transluminal percutaneous angioplasty in cases of thrombosis and venous stenosis. In addition, the use of prostheses is of great importance in the management of cases utilization of traumatic lesion to the SVC during lung or mediastinal cancer surgeries. In the realm of radiotherapy, the fractionating technique allows for the administration of high doses of irradiation to cancer patients with benefits to $70 \%$ of the cases on the average.

Descritores - Veia cava superior. Tomografia computadorizada por raios X. Ressonância magnética nuclear. Neoplasias pulmonares. Key words - Superior vena cava. CT scan with X-rays. Magnetic resonance. Lung neoplasms.

* Trabalho realizado no Serviço de Tórax do Hospital do Câncer, Instituto Nacional de Câncer, Ministério da Saúde, Rio de Janeiro, RJ.

1. Médico Cirurgião do Serviço de Tórax. Doutor em Cirurgia Torácica.

2. Médico Cirurgião do Serviço de Tórax. Professor Titular de Cirurgia Torácica.

Endereço para correspondência - E-mail: samuelcirtorax@zipmail. com.br. Tel. (21) 2712-8295; fax (21) 2719-9204.

Recebido para publicação em 26/7/02. Aprovado, após revisão, em 20/8/02.
Siglas e abreviaturas utilizadas neste trabalho

VCS - Veia cava superior

SVCS - Síndrome de veia cava superior

PTFE - Politetrafluoroetileno

TC - Tomografia computadorizada

RM - Ressonância magnética

\section{HISTÓRICO}

A svCS tem a sua descrição inicialmente relacionada a uma época em que não se conheciam os antibióticos. Em 1757, o primeiro registro de paciente com síndrome era publicado por William Hunter e se referia a portador de sífilis que desenvolvera aneurisma sifilítico da aorta torá- 


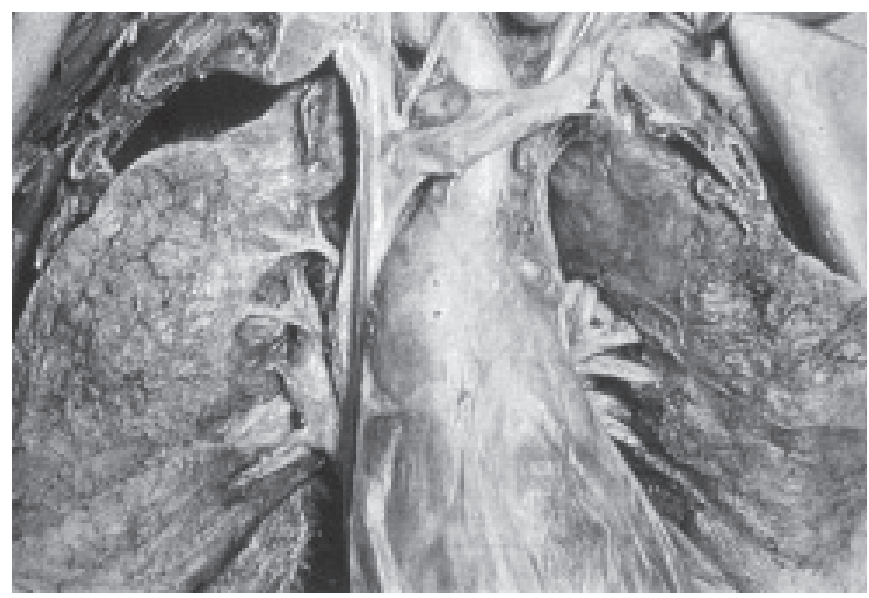

Figura 1 - Anatomia mostrando a VCS e suas relações no mediastino

cica(1). Doenças infecciosas como a sífilis e a tuberculose mediastinal foram as causas de $40 \%$ dos casos na série de 274 revistos e publicados por Schedter em 1954. Atualmente, o carcinoma de pulmão é responsável por $70 \%$ dos casos, as doenças malignas do mediastino e fibroses mediastinais não malignas assim como tromboses relacionadas a cateteres são a maioria das causas restantes. Hoje se sabe que não apenas $5 \%$ das causas são de natureza benigna, há incidência maior, ainda não bem estabelecida em percentuais ${ }^{(2)}$.

\section{SINAIS E SINTOMAS}

A SVCS, por definição, é a expressão clínica da obstrução ao fluxo sanguíneo na VCS. A síndrome clínica tem uma gradação nas manifestações que é correlata ao grau de obstrução da veia no mediastino ${ }^{(3)}$. Detectar os sinais iniciais da síndrome como o edema facial matutino é tarefa diária do pneumologista ou cirurgião torácico; constitui um dado importante na elaboração do diagnóstico final que pode incluir as punções aspirativas, biópsias linfonodais e mediastinais (Figuras 1 e 2).

A dispnéia, a pletora facial e o edema cervicofacial são os sintomas mais freqüentes e até $60 \%$ dos pacientes chegam aos consultórios com estas queixas ${ }^{(3-5)}$. Podem aparecer tosse, edema dos membros superiores, dor torácica e disfagia. Os sinais e sintomas mais freqüentemente encontrados e sua gradação evolutiva estão representados no Quadro 1.

A tosse-síncope chama a atenção por ser algumas vezes a primeira manifestação da síndrome. O relato do paciente é de perda súbita da consciência durante uma crise de tosse. Sua etiopatogenia é explicada pela equiparação das pressões nos capilares arterial e venoso junto à substância cerebral, a pressão no sistema venoso está elevada (200 a $500 \mathrm{~cm}$ de água) e se eleva ainda mais du-

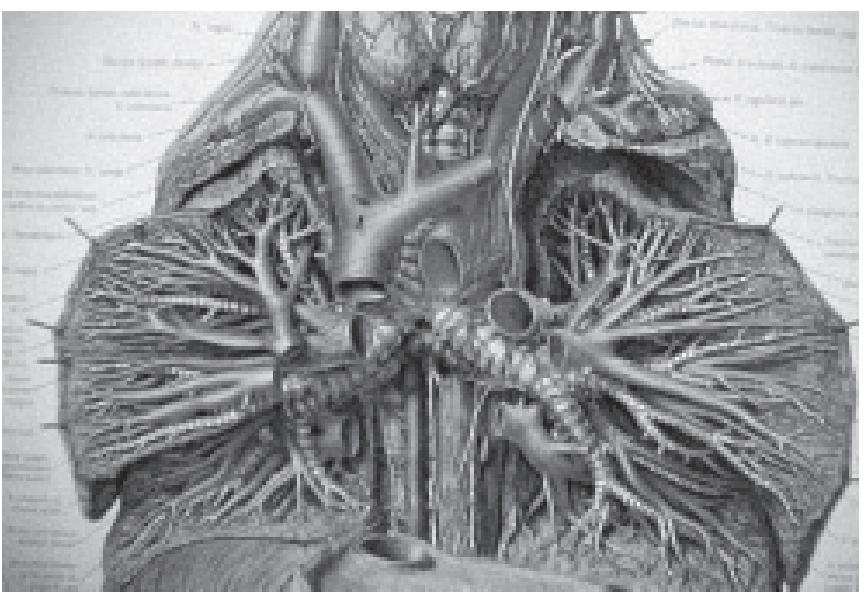

Figura 2 - Ilustração anatômica do mediastino com VCS e suas relações após remoção do coração. (Retirado de Atlas de Anatomia Humana, Pernkopf, 1980).

\begin{tabular}{|ll|}
\hline \multicolumn{1}{|c|}{ QUADRO 1} \\
Sinais, sintomas e evolução da síndrome da veia cava superior \\
\hline Compressão/obstrução - Inicial & - Edema facial matutino \\
$60 \%$ dos casos & - Edema cervicofacial \\
& - Pletora facial \\
& - Dispnéia \\
& - Turgência venosa cervical \\
\hline Edema peribrônquico & - Edema membros superiores \\
e periesofagiano & - Dor torácica \\
& - Disfagia \\
& - Turgência venosa em \\
& - Tosse-síncope \\
\hline Edema cerebral - & - Obnubira \\
Sintomas neurológicos & - Coma \\
\hline Obstrução completa & - pirculação colateral torácica \\
\hline
\end{tabular}

rante a tosse. Ocorrem isquemia transitória e síncope. Alguns pacientes, mesmo depois de tratados, podem apresentar episódios semelhantes até que a circulação colateral alivie a hipertensão venosa.

$\mathrm{O}$ aparecimento de circulação colateral é dependente do grau de obstrução da VCS, fica evidente na fase de oclusão completa do vaso quando as veias subcutâneas se pronunciam e mostram aspecto varicoso à parede anterior do tórax, as telangiectasias são freqüentemente vistas também neste local. As veias ázigos, torácicas internas ou mamárias, torácicas laterais, assim como as veias paraespinhais e o plexo venoso periesofagiano também se engurgitam na tentativa de encontrar um caminho de 
retorno do sangue das extremidades superiores para o coração. A pressão nestes vasos se eleva porque a drenagem venosa está prejudicada. Ao estabelecer acesso venoso para infusão de soluções, as veias escolhidas devem ser as dos membros inferiores. As infusões nas veias dos membros superiores ou cervicais podem piorar o quadro clínico. O edema peribrônquico e o edema cerebral são responsáveis por esta piora(6).

Os sintomas neurológicos são relacionados ao grau de edema cerebral existente. Podem aparecer as síncopes após tosse, as tonteiras, a cefaléia e a sensação de pletora facial. O estado de coma pode instalar-se e ser aliviado pela mudança da via de acesso venoso, pelo uso de diuréticos e corticosteróides. A elevação da cabeceira do leito e o enriquecimento do ar inspirado por oxigênio úmido são medidas que auxiliam no tratamento.

O edema intermitente das extremidades superiores $e$ inferiores e também o da parede abdominal podem ser relatados, podem estar relacionados a uma estenose membranosa das veias cava superior e inferior.

As tromboflebites de VCS podem resultar em oclusão do vaso com SVCS; a candidíase através de cateter venoso implantado deve ser investigada ${ }^{(7)}$. A retirada imediata do cateter mediante heparinização e a semeadura de sua ponta para as culturas podem identificar o agente causal.

\section{Diagnóstico}

O diagnóstico de SVCS deve ser estabelecido mediante a história e o exame físico. É de importância fundamental valorizar queixas como "levantar de manhã com o rosto inchado", e edemas ou hiperemias cervicofaciais sem outras explicações, o encontro de ingurgitamento venoso cervical e a presença de circulação colateral torácica, o relato de perda da consciência relacionada ao esforço de tosse ou esforço físico maior ${ }^{(8,9)}$.

Estabelecido o diagnóstico clínico, os exames complementares deverão ser realizados incluindo as telerradio-

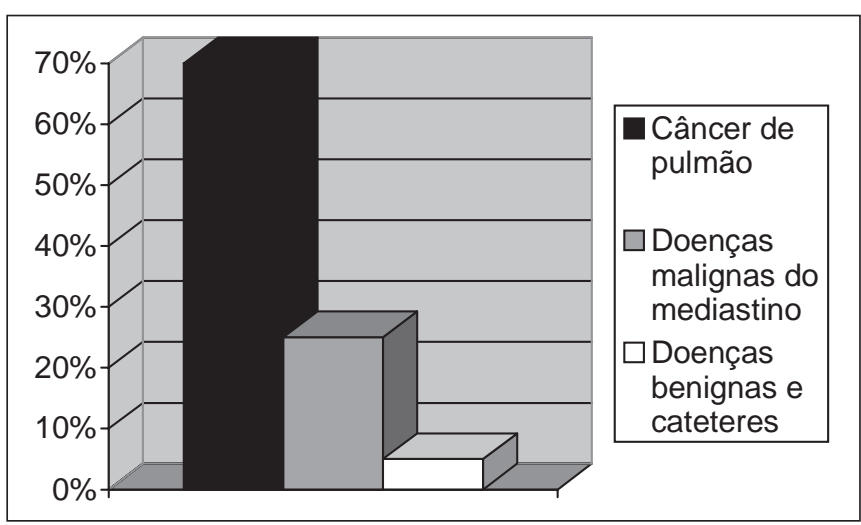

Figura 3 - Principais causas da SVCS expressas em grupos de doenças grafias de tórax em PA e perfil, as tomografias computadorizadas ou a ressonância magnética. Os exames de imagem podem apontar inicialmente o alargamento mediastinal devido à presença de massa ou linfonodomegalia. O uso do contraste venoso deve mostrar claramente o nível da obstrução. A localização pode estar na junção dos troncos braquiocefálicos, nas veias subclávias, nas jugulares ou na própria VCS. A presença de trombo intraatrial deve ser investigada. A cavografia (Figuras 4 e 5) não tem sido utilizada porque a tomografia contrastada a substitui com vantagens (visão em cortes transversos $e$ planos) $)^{(10)}$

A presença de linfonodos calcificados no mediastino aponta para o diagnóstico de histoplasmose. Mediastino aparentemente normal na radiografia de tórax inicial não

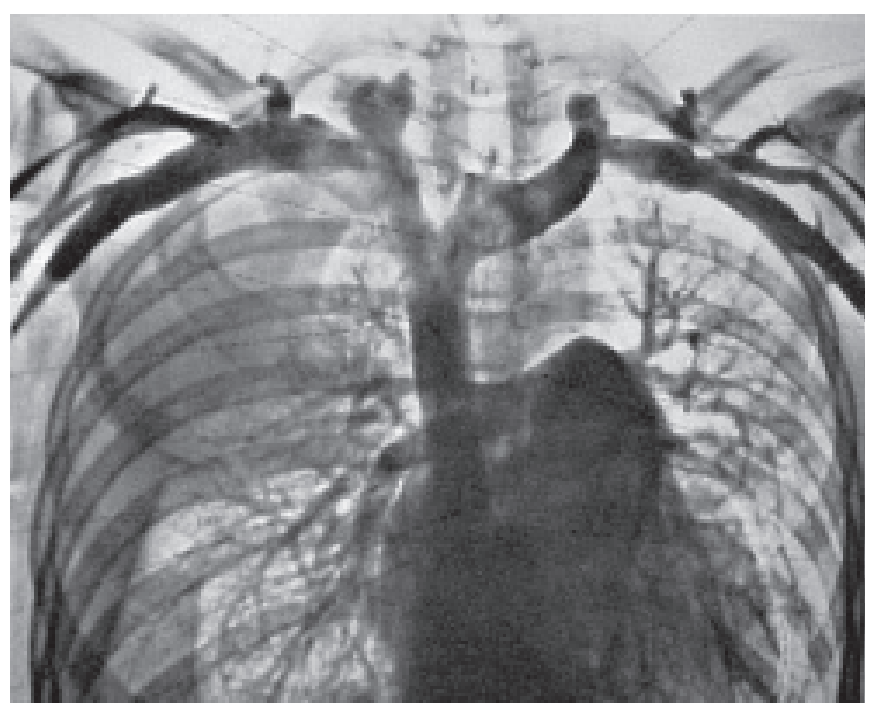

Figura 4 - Cavografia superior normal numa angiocardiografia feita em paciente com derrame pleural direito. (Retirado de Atlas de Anatomia Humana, Pernkopf, 1980).

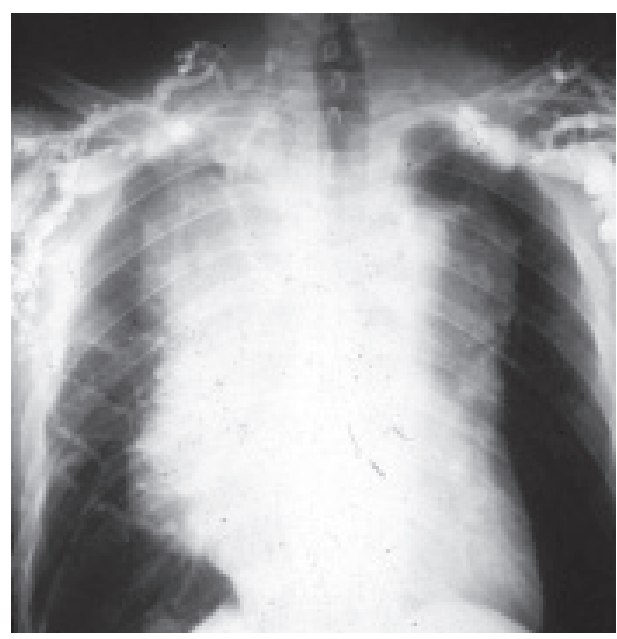

Figura 5 Cavografia superior mostrando obstrução do fluxo de contraste venoso ao nível das veias subclávias. Caso de paciente portador de seminoma e SVCS. 


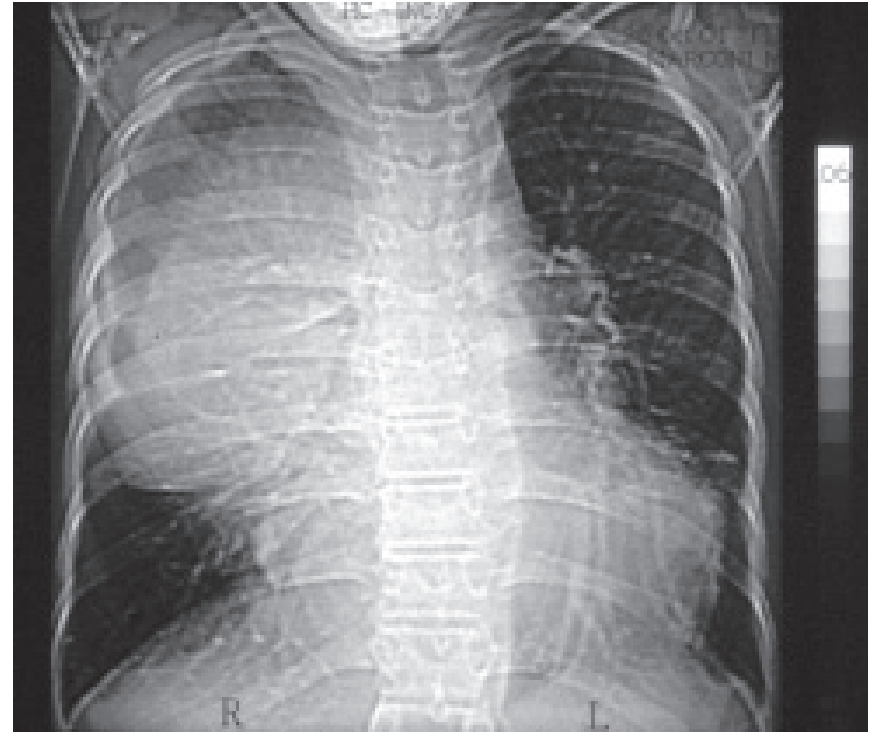

Figura 6 - Radiografia de tórax em PA, opacidade devida à massa mediastinal. Compressão de VCS por teratoma.

\begin{tabular}{|ll|}
\hline \multicolumn{1}{|c|}{$\begin{array}{c}\text { QUADRO } 2 \\
\text { Achados radiológicos na SVCS }\end{array}$} \\
\hline Radiografia de tórax & - Aspecto normal \\
Mediastino/hilo do pulmão & - Alargamento \\
& - Calcificações \\
& - Massa \\
& - Linfonodomegalia \\
\hline TC contrastada de tórax & - Obstruções venosas \\
RM & - Compressão extrínseca \\
& - Invasão \\
& Envolvimento $>180^{\circ}$ \\
& da circunferência do vaso \\
\hline
\end{tabular}

exclui a fibrose mediastinal ou a estenose membranosa como causa de $\operatorname{SVCS}^{(11,12)}$.

Os tumores e massas mediastinais deverão ser estudados quanto à relação com a VCS, se há compressão extrínseca (Figuras 6 e 7), invasão mínima, invasão parcial ou total do vaso. Estes dados são de importância fundamental na decisão da operabilidade e ressecabilidade. A simples compressão do vaso não contra-indica cirurgia de ressecção no câncer de pulmão. O envolvimento da circunferência do vaso maior que $180^{\circ}$ pela massa mostra um critério de exclusão para ressecção radical(13). Os achados radiológicos mais freqüentemente encontrados na SVCS estão representados no Quadro 2.

O uso do Doppler é de grande valor no pós-operatório de pacientes que receberam circulação extracorpórea porque pode identificar tromboses e ser realizado à beira do leito ${ }^{(14)}$.

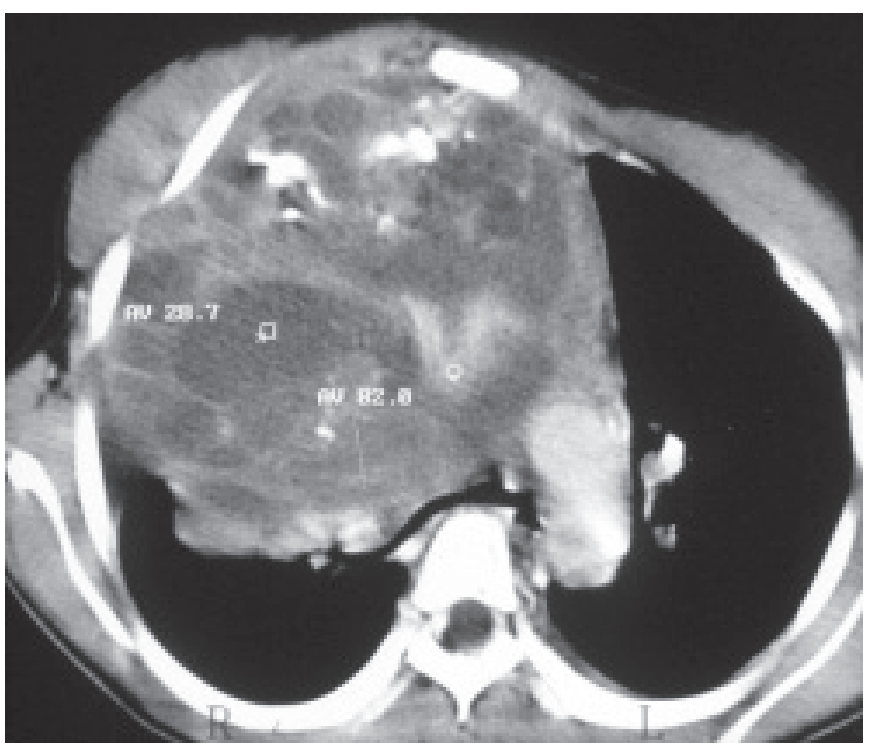

Figura 7 - TC de tórax. Compressão de VCS de etiologia benigna. Teratoma operado, tendo sido aliviada a compressão após ressecção da massa.

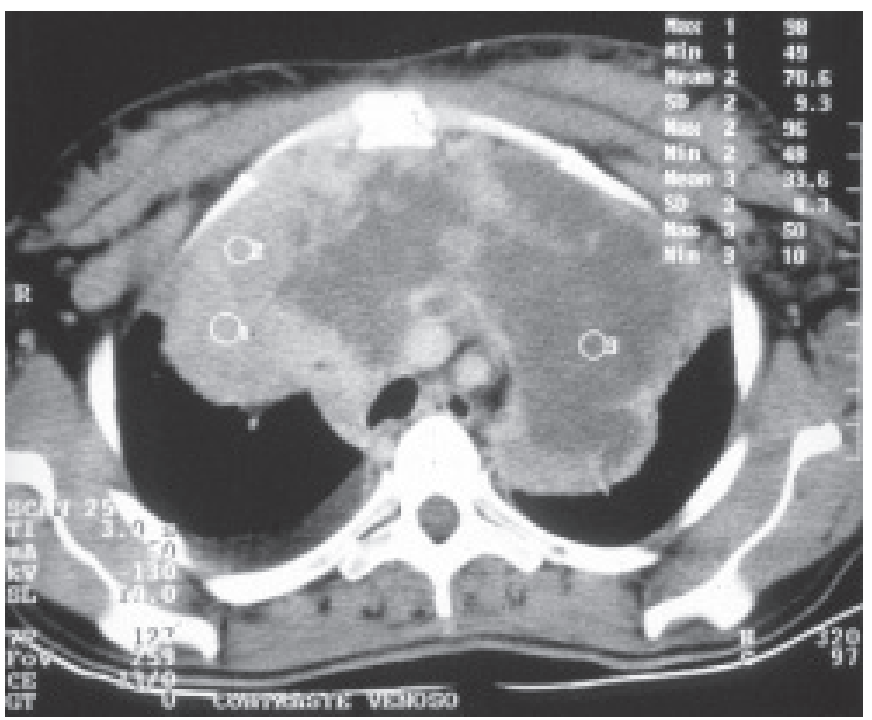

Figura 8 - TC de tórax. Caso de linfoma em paciente com SVCS.

As punções aspirativas são realizadas sobre as massas na SVCS, preferencialmente na região paraesternal direita. As massas mediastinais que não forem definidas pela citopatologia podem requerer mediastinoscopia para biópsia (Figura 8).

O estabelecimento do diagnóstico histopatológico das massas mediastinais pode requerer o uso da mediastinoscopia ou a mediastinotomia paraesternal mesmo na presença de SVCS. O procedimento tem sido utilizado sem que haja complicações hemorrágicas; a circulação colate- 
ral mais acentuada está no subcutâneo e não sob a fáscia pré-traqueal onde a dissecção é realizada.

Os linfonodos localizados nas fossas supraclaviculares assim como os nódulos subcutâneos de aparecimento recente podem revelar o diagnóstico.

\section{ETIOLOGIA}

O câncer do pulmão é responsável por $70 \%$ dos casos de SVCS (Figura 3). De uma série de 4.100 casos de câncer do pulmão relatada na literatura, $2,4 \%$ dos pacientes apresentavam a SVCS. O carcinoma indiferenciado de pequenas células é o tipo histológico mais comum, aparecendo em até $38 \%$ dos casos ${ }^{(15,16)}$. Os linfomas acrescentam 8\% dos casos de SVCS, sendo o tipo esclerótico o mais freqüente. O linfoma de Hodgkin envolve freqüentemente o mediastino, mas raramente causa a síndrome $e^{(17)}$. Timomas e tumores de células germinativas podem ser a causa. A leucemia é uma causa rara desta síndrome. $\mathrm{O}$ carcinoma metastático de mama é o mais freqüente entre as causas neoplásicas secundárias. Entre as causas não malignas, a fibrose mediastinal secundária à histoplasmose parece ser a mais freqüente. O bócio subesternal de grandes proporções pode manifestar-se por SVCS; a compressão da veia no espaço mediastinal entre a traquéia $e$ o esterno leva a essa situação(18,19). Os cateteres implantados no sistema cava superior assim como o Swan-Ganz e também os marca-passos podem levar a trombose $e$ $\mathrm{SVCS}^{(20)}$. Lesões mais raras como o hemangioendotelioma e a doença de Behçet podem causar oclusão da VCS.

As doenças granulomatosas do mediastino podem causar SVCS por aumento do tamanho dos linfonodos mediastinais e compressão da veia(21,22).

\section{TRATAMENTO}

O tratamento clássico da SVCS inclui o uso de medidas clínicas como elevação da cabeceira do leito, oxigênio, diuréticos e corticosteróides, visa diminuir o edema no território drenado pela VCS e reduzir assim os sintomas neurológicos e respiratórios presentes nessa síndrome. $\mathrm{O}$ uso de via venosa apenas em membros inferiores serve para evitar não só a piora dos sintomas da hipertensão venosa, mas, também, que drogas injetadas demorem mais tempo do que o habitual para ser distribuídas na circulação sistêmica(23).

O tratamento da síndrome requer o estabelecimento do diagnostico etiológico; nem sempre as condições clínicas dos pacientes são as melhores e o tratamento medicamentoso inicial é necessário para obter condições razoáveis.

Hoje sabemos que os avanços na área da citopatologia e no diagnóstico histopatológico dos linfomas e massas mediastinais, associados à melhora clínica com a abordagem medicamentosa, evitam que se trate com irradiações uma SVCS de origem não maligna. Portanto, procedemos ao estabelecimento do diagnóstico cito ou histopatológico antes da terapêutica específica no caso de tumores.

A indicação de cirurgia de enxerto na SVCS de etiologia maligna tem suas limitações porque a sobrevida não é alargada após este procedimento. Algumas situações específicas, como nos casos de timomas invasivos tratados inicialmente por quimioterapia e/ou radioterapia, podem ter na cirurgia do enxerto um recurso para a ressecção de toda lesão inicial. Já tivemos pacientes em que o resíduo tumoral junto à VCS foi ressecado em bloco com o resquício tumoral sem que a VCS precisasse ser substituída. No caso de $\mathrm{N}_{2}$ do câncer de pulmão não há indicação para cirurgia de enxerto. Nos casos de $\mathrm{N}_{0}$ ou $\mathrm{N}_{1}$ da doença, a extensão do tumor com compressão da VCS permite que se complete a ressecção com substituição da $\mathrm{VC}$, se necessário. A mortalidade pós-operatória é baixa e pode significar a cura.

A ressecção dos tumores mediastinais e a substituição da VCS só estão indicadas quando curativas. Cirurgia paliativa não deve ser realizada devido ao grande porte $e$ riscos inerentes ${ }^{(24)}$. Na presença de circulação colateral extensa e desenvolvida, é contra-indicada a cirurgia de enxerto pelo risco de tromboembolismo.

O enxerto de melhor aceitação é o realizado entre a veia inominada ou jugular do lado esquerdo $e$ a auriculeta direita, utilizando uma prótese em anastomose términoterminal. A prótese de uso mais comum é de PTFE (politetrafluoroetileno). A prótese é suturada inicialmente junto ao átrio e depois à veia superior escolhida, permitindo que todo o ar do sistema seja extravasado por esta última sutura antes que o clampeamento superior seja retirado. O PTFE de número 18 ou 20, devidamente heparinizado, assim como no pós-operatório a anticoagulação deve ser mantida. Cuidados com a monitorização e manutenção da pressão venosa central evitam tromboses ${ }^{(24,25)}$.

Outras técnicas incluem auto-enxerto com pericárdio e enxerto em espiral utilizando a veia safena autóloga, menos utilizados que o primeiro por requerer mais tempo em sua elaboração(26).

A angioplastia percutânea transluminal é utilizada para as obstruções trombóticas induzidas por cateter; é realizada através de inserção de stent metálico expansível ou de balões infláveis e capazes de abrir a luz da VCS, mantendo assim sua permeabilidade. O Wallstent ${ }^{(27)}$ e o Gianturco $Z$ stent são utilizados e podem oferecer até $93 \%$ de respostas sintomáticas ${ }^{(28)}$.

A heparina seguida pelos anticoagulantes orais pode reduzir a extensão do trombo e prevenir sua progressão. A remoção do cateter combinada com anticoagulação é outra alternativa viável(29). 
Nos pacientes com diagnóstico estabelecido de carcinoma de pulmão não pequenas células, o tratamento pelas irradiações deve ser o indicado nos casos de SVCS. O alívio sintomático é freqüente e alguns pacientes chegam a dois anos de sobrevida (apenas 2\%); a sobrevida de um ano após as irradiações pode chegar aos 17\%. Portanto, não é um prognóstico favorável, mas a qualidade de vida melhora com esse tratamento. O esquema fracionado de irradiação é o mais utilizado. Inclui duas a quatro frações iniciais de 300 a $400 c G y$, seguidos por um fracionamento convencional até uma dose total de 3.000 a $5.000 \mathrm{cGy}$. Há melhora clínica de $70 \%$ dos pacientes com esse tipo de esquema ${ }^{(30,31)}$.

\section{REFERÊNCIAS}

1. Hunter W. The history of an aneurysm of the aorta with some remarks on aneurysms in general. Med Observ Inq 1757; 1:323.

2. De las Heras Garcia B, Jimenez Gordo A, Casado Saenz E, Zamora Aunon P, Cantalejo Moreira M, Gonzalez Baron M. Superior vena cava syndrome: presentation of six cases. An Med Interna 2001;18: 369-72.

3. Baumgarten AS, Richardson C, Dolan D. Superior vena cava syndrome. J Fam Pract 1986;23:375-6.

4. Shimada T, Kitamura J. Superior vena cava syndrome. Ryoikibetsu Shokogun Shirizu 1996;14:497-501.

5. Sheppard KC. Care of the patient with superior vena cava syndrome. Heart Lung 1986;15:636-41.

6. Vazhenin AV, Kotliarov EV, Abdulina NA. Diagnosis and treatment of the superior vena cava syndrome. Klin Med (Mosk) 1988;66:43-5.

7. Haro M, Coloma R, Arevalo M. The superior vena cava syndrome and juxtamediastinal bullae. Arch Bronconeumol 1995;31:255-6.

8. Frank AR. Superior vena cava syndrome. Current management concepts. Nebr Med J 1989;74:8-16.

9. Villacastin BP, Robledo AG, Nunez A, Miro C, Franco A, de Sequera $P$, et al. Superior vena cava syndrome: radiologic study. Rev Clin Esp 1991;189:293-5.

10. Huang JS, Liaw CC, Ng KT, Shih LY, Lee CH, Lin FJ. Superior vena cava syndrome clinical analysis of 92 cases. Changgeng Yi Xue Za Zhi 1988;20;11:107-15.

11. Kovacs RG, Aguayo SM. Images in clinical medicine. Superior vena cava syndrome. N Engl J Med 1993;30:1007.

12. Brown NJ. A woman with superior vena cava syndrome. J Tenn Med Assoc 1992;85:329-30.

13. Perez-Segura P, Colmenarejo Rubio A. Syndrome of the superior vena cava (Compilation of the cases of the San Carlos University Clinical Hospital in Madrid in the last 5 years and review of the literature). An Med Interna 1995;12:560-4.
14. Montgomery JH, D'Souza VJ, Dyer RB, Formanek AG, Prabhu SH. Nonsurgical treatment of the superior vena cava syndrome. Am J Cardiol 1985;56:829-30.

15. Urban T, Lebean B, Chastang C. Superior vena cava syndrome in small cell lung cancer. Arch Intern Med 1993;153:384.

16. Wurschmidt F, Bunemann H, Heilmann HP. Small cell lung cancer with and without superior vena cava syndrome: a multivariate analysis of prognostic factors in 408 cases. Int J Radiat Oncol Biol Phys 1995; $33: 77$.

17. Lazzarinni M, Orlandi E, Paulli M. Primary mediastinal B cell lymphoma with sclerosis: an aggressive tumor with distinctive clinical and pathologic features. J Clin Oncol 1993;11:2306.

18. Nieto AF, Doty DB. Superior vena cava obstruction: clinical syndrome, etiology and treatment. Curr Probl Cancer 1986;10:441-84.

19. Fitt AW, Rauz S, Kirkby GR. Obstruction of the superior vena cava. Br J Ophthalmol 1998;82:1092

20. Brullet E, Nogureras A, Cantarell G, Ripoll E. Superior vena cava syndrome and pacemaker. Rev Esp Cardiol 1986;39:317-8.

21. Musumeci ML, De Pasquale R, Tedeschi A, Neri S, Micali G. Non malignant superior vena cava syndrome. Int J Dermatol 2000;39:9346.

22. Park JY, Chung-Park M, Snow M. Intravascular papillary endothelial hyperplasia of superior vena cava: a rare cause of the superior vena cava syndrome. Thorax 1991;46:272-3.

23. Biglioli P, Spirito R, Bacciu PP, Sala A, Susini G, Antona C. Our experience with the treatment of obstruction of the superior vena cava. Ann Chir 1985;39:500-7.

24. Dartevell PG, Pastorino U, Lenot B, Bavoux EA, Neveux JY, Chapelier AR. Long-term follow-up after prosthetic replacement of the superior vena cava combined with resection of mediastinal pulmonary malignant tumors. J Thorac Cardiovasc Surg 1991;102:259-65.

25. Cordeiro PB. Síndrome de veia cava superior [tese]. Niterói: Faculdade de Medicina, Universidade Federal Fluminense, 1985.

26. Doty DB. Bypass of superior vena cava. Six years' experience with spiral vein graft for obstruction of VCS. J Thorac Cardiovasc Surg 1982;83:326.

27. Hennequin LM, Fade O, Fays JG. Superior vena cava stent placement: results with the wallstent endoprosthesis. Radiology 1995;196: 353.

28. Sundersk, Ekong EA, Sivalingam K, Kumar A. Superior vena cava thrombosis due to pacing electrodes: successfull treatment with combined thrombolysis and angioplasty. Am Heart J 1992;123:790.

29. Hansen M, Garsdal P. The superior vena cava syndrome caused by a transvenous cardiac pacemaker. Ugeskr Laeger 1985;12;147:2614.

30. Torras Rosello J, Ripol Girona S. Indications for radiotherapy in the superior vena cava syndrome. Rev Esp Oncol 1985;32:543-50.

31. Yellin A, Rosen A, Reichert N, Lieberman Y. Superior vena cava syndrome: the myth, the facts. Am Rev Respir Dis 1990;141:1114. In De Vita VT, editor. Oncologic emergencie. $5^{\text {th }}$ ed. Philadelphia: Lippincott Raven Publishers, 1997. 